\section{Moving towards early clinical trials for amyloid-targeted therapy in Alzheimer's disease}

\author{
Paul S. Aisen, Bruno Vellas and Harald Hampel
}

A recent article in Nature Reviews Drug Discovery (Nature Rev. Drug Discov. 11, 657-660; 2012 $)^{1}$ highlighted the outcome of the two largest Alzheimer's disease drug development programmes to date. Results from the Phase III clinical trials of two monoclonal antibodies - bapineuzumab and solanezumab - that target amyloid- $\beta$ indicated little clinical benefit of immunological attack on amyloid- $\beta$ at the dementia stage of sporadic disease ${ }^{2}$ (see the 12 December 2012 press release on the Lilly website).

Neither antibody demonstrated a favourable impact on the primary cognitive assessment scores and inventories of daily function in patients with mild to moderate Alzheimer's disease-related dementia. However, planned post-hoc pooled analyses of data from individuals with mild Alzheimer's disease-related dementia treated with solanezumab did show cognitive benefit, so the development of this agent for the treatment of Alzheimer's-related dementia will continue with a confirmatory clinical trial planned for launch later this year (See the 12 December 2012 press release on the Lilly website).
The negative primary analyses in these two programmes represent the culmination of a 'lost decade' in Alzheimer's disease therapeutic trials, with no substantial success since the approval of memantine. Studies of hypothesis-driven candidate disease modifiers such as anti-inflammatory drugs, secretase inhibitors and modulators, hormonal therapies, statins and other drugs have been disappointing. The clinical failure of the two leading $\gamma$-secretase inhibitors, semagacestat (see the 17 August 2010 press release on the Lilly website) and avagacestat (see the Bristol-Myers Squibb website), have added further gloom to the outlook.

The major approach to disease modification has been the targeting of amyloid- $\beta$ accumulation in the brain. Genetic evidence strongly indicates that amyloid- $\beta$ can drive the disease process, so reducing its production or promoting its clearance is an attractive aim ${ }^{3}$. Although accumulation of amyloid- $\beta$ in the brain is probably multifactorial in late-onset sporadic Alzheimer's disease (in contrast to autosomal dominantly inherited early-onset Alzheimer's disease), it is plausible that amyloid-targeted
Table 1 | Characteristics of an early (asymptomatic) Alzheimer's disease trial*

\begin{tabular}{ll} 
Characteristic & Details \\
Population & $\begin{array}{l}\text { - Sporadic Alzheimer's disease: amyloid- } \beta \text {-positive above } 70 \text { years of age } \\
\text { - Familial Alzheimer's disease: mutation carriers } 10 \text { years prior to expected } \\
\text { onset of symptoms } \\
\text { - Down's syndrome: above } 45 \text { years of age }\end{array}$ \\
\hline Intervention & $\begin{array}{l}\text { Active or passive amyloid-targeted immunotherapy } \\
\text { - } \gamma \text {-secretase inhibitor or } \beta \text {-secretase inhibitor } \\
\text { - Tau-directed therapy } \\
\text { - Neuroprotectant }\end{array}$ \\
\hline - Combination therapy
\end{tabular}

CSF, cerebral spinal fluid, MRI, magnetic resonance imaging, PET, positron emission tomography.

*Also referred to as a secondary prevention trial. interventions may yield clinical benefit in all forms of Alzheimer's disease if they are initiated very early, before severe synaptic dysfunction and irreversible widespread cell loss and neurodegeneration have occurred ${ }^{4}$. Indeed, the recent discovery that a genetically determined reduction in amyloid- $\beta$ peptide production by $40 \%$ provides dramatic protection against Alzheimer's disease $\mathrm{e}^{5}$ further suggests that the clinical failures may relate primarily to the timing of the intervention.

Consensus regarding the pathophysiological processes that underlie Alzheimer's disease progression is reflected by the newly revised criteria for diagnosing Alzheimer's disease-related dementia, mild cognitive impairment and preclinical disease ${ }^{6}$. In particular, the concept of a biomarker-defined pre-symptomatic stage of Alzheimer's disease provides a foundation for earlystage clinical trials. Based on the prevailing view that the accumulation of amyloid- $\beta$ in the brain is a principal inciting event in Alzheimer's disease pathophysiology, this early stage of the disease can be recognized by abnormal amyloid positron emission tomography (PET) scans or a reduction in the levels of amyloid peptides in cerebrospinal fluid.

Although at this stage individuals are normal from a clinical perspective, they can be accurately identified and disease progression can be tracked using dynamic biochemical and imaging biomarkers as well as tests of cognitive performance $e^{7,8}$. Although it remains to be proven whether all individuals with brain amyloidosis will progress to symptomatic Alzheimer's disease if they live sufficiently long, this is a plausible hypothesis that is consistent with the available autopsy and biomarker data ${ }^{9}$. Indeed, analyses of data from multiple studies indicate that asymptomatic individuals with amyloid- $\beta$ accumulation in the brain show faster cognitive decline than similar individuals without amyloidosis.

Indeed, Alzheimer's disease progression may involve a long, symptom-free phase preceding gradual cognitive, functional and behavioural decline that is functionally adaptive and plausibly reversible. Therefore, efforts to develop disease-modifying treatments may require clinical trials to be conducted much earlier in the disease process. Studies can be powered based on the hypothesis that amyloid-targeted or other disease-modifying interventions can reduce the rate of amyloid-mediated decline towards normal trajectories. It is possible that regulatory authorities the in United 


\section{a Cognitive composite}

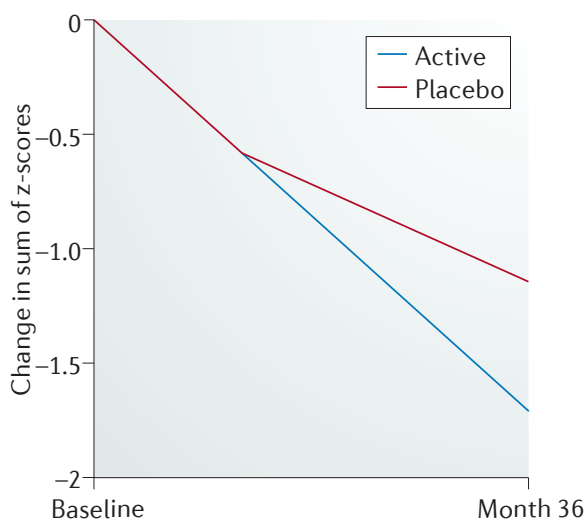

b CSF p-tau

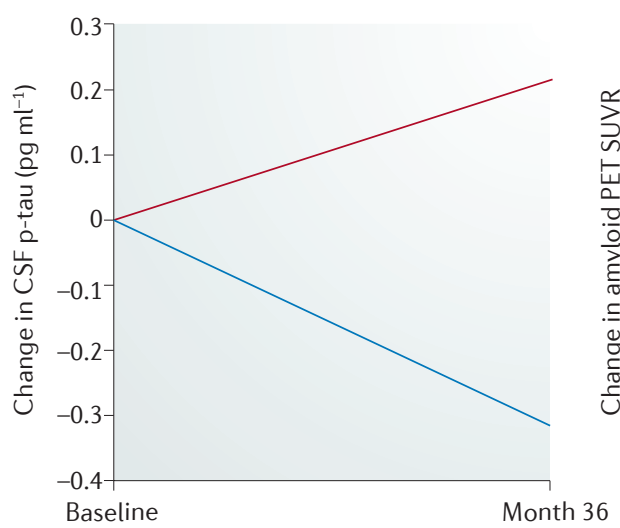

c Amyloid PET

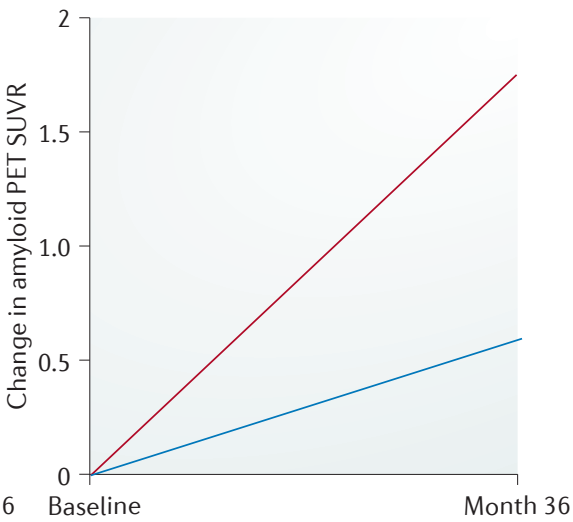

Figure 1 | Hypothetical results of an early Alzheimer's disease clinical trial. Hypothetical results are shown of a randomized clinical trial that compares placebo to an active treatment with an agent that reduces amyloid- $\beta$ levels over 36 months, which might lead to regulatory approval (with requirement for a postmarketing study to confirm clinical benefit). a|The change in $z$-score is shown for a composite cognitive assessment that includes measures of episodic memory and executive function. $\mathbf{b}$ |The change in cerebrospinal fluid (CSF) levels of phosphorylated tau ( $p$-tau) is shown. c| The change in the composite standardized uptake value ratio (SUVR) measured by amyloid positron emission tomography (PET) scan is shown.
States and Europe could approve a drug for the treatment of preclinical Alzheimer's disease on the basis of a single, primary cognitive end point that is supplemented by a panel of biomarkers and postmarketing studies to support clinical benefit (TABLE 1; FIG. 1); see the US Food and Drug Administration (FDA)'s draft guidance. This contrasts with the current requirement for cognitive assessments combined with functional and/or global assessments.

Using these principles, three early-stage trials will launch within the next year, one in patients with sporadic disease (see the article titled "NIH-supported Alzheimer's studies to focus on innovative treatments" on the US National Institutes of Health website) and two in families with mutations associated with autosomal dominant Alzheimer's disease (ClinicalTrials.gov identifier: NCT01760005; see the 21 May 2012 news article on the Alzheimer Research Forum). These clinical trials will use a composite cognitive end point. In addition, a pilot longitudinal biomarker study in individuals with Down's syndrome is planned, as a foundation to clinical trials in this group of patients who are at a high risk of developing Alzheimer's disease ${ }^{10}$.

It is more likely that these secondary prevention trials, rather than the recent dementia-stage trials, will demonstrate the beneficial effects of anti-amyloid therapy on disease progression. But it is plausible that the most effective intervention will be primary prevention in individuals at risk (by virtue of age, genetics and/or other factors) but without biomarker-based evidence of Alzheimer's disease neurobiology. Therefore, additional collaborative biomarker studies are required to inform the design of such clinical trials. Our long-term vision includes risk factor and biomarker monitoring of the ageing population, with the use of effective anti-amyloid (and perhaps neuroprotective) therapies that will succeed in quelling the Alzheimer's disease epidemic. Recent trial results have been disappointing, but we find cause for optimism.

Paul S. Aisen is at the Department of Neurosciences, University of California, San Diego, 9500 Gilman Drive M/C 0949, San Diego, California 92093, USA.

Bruno Vellas is at the Department of Internal Medicine and Geriatrics, Toulouse University Hospital, 170 Avenue de Casselardit, TSA 40031 31059 Toulouse Cédex 9, France.

Harald Hampel is at the Department of Psychiatry, University of Frankfurt, Heinrich-Hoffman-Str. 10, 60528 Frankfurt, Germany.

Correspondence to P.S.A. e-mail:paisen@ucsd.edu doi: 10.1038/nrd3842-c1 Published online 15 March 2013

1. Mullard, A. Sting of Alzheimer's failures offset by upcoming prevention trials. Nature Rev. Drug Discov. 11, 657-660 (2012).
2. Sperling, R. et al. A randomized, double-blind placebo controlled clinical trial of intravenous bapineuzumab in patients with mild to moderate Alzheimer's disease who are apolipoprotein E $\varepsilon 4$ carriers. European Federation of Neurological Societies [online], http://www2.kenes.com/efns/info/Documents/Sperling Bapineuzumab\%20IV\%20Study \%20302_EFNS\%20 Presentation\%20Slides 9-11-2012.pdf (Stockholm, Sweden; 2012)

3. Hardy, J. \& Selkoe, D. J. The amyloid hypothesis of Alzheimer's disease: progress and problems on the road to therapeutics. Science 297, 353-356 (2002).

4. Sperling, R. A., Jack, C. R. \& Aisen, P. S Testing the right target and right drug at the right stage. Sci. Transl. Med. 3,111 cm33 (2011).

5. Jonsson, T. et al. A mutation in APP protects against Alzheimer's disease and age-related cognitive decline. Nature 488, 96-99 (2012).

6. Sperling, R. A. et al. Toward defining the preclinical stages of Alzheimer's disease: recommendations from the National Institute on Aging-Alzheimer's Association workgroups on diagnostic guidelines for Alzheimer's disease. Alzheimers Dement. 7, 280-292 (2011).

7. Hampel, H. et al. Biomarkers for Alzheimer's disease: academic, industry and regulatory perspectives. Nature Rev. Drug Discov. 9, 560-574 (2010).

8. Bateman, R. J. et al. Clinical and biomarker changes in dominantly inherited Alzheimer's disease; the Dominantly Inherited Alzheimer Network. N. Engl. J. Med. 367, 795-804 (2012)

9. Rowe, C. C. et al. Amyloid imaging results from the Australian Imaging, Biomarkers and Lifestyle (AIBL) study of aging. Neurobiol. Aging. 31, 1275-1283 (2010).

10. Ness, S. et al. Down's syndrome and Alzheimer's disease: towards secondary prevention. Nature Rev. Drug Discov. 11, 655-656 (2012).

\section{Acknowledgements}

P.S.A.'s work is supported by grants from the US National Institute on Aging of the US National Institutes of Health. B.V.'s work is supported by Gerontopole, CHU, INSERM UMR 1027, University of Toulouse. H.H.'s work is supported by grants from the Katharina-Hardt-Foundation, Bad Homburg, Germany. 